\title{
Update on the secondary cytoreduction in platinum-sensitive recurrent ovarian cancer: a narrative review
}

\author{
Carmine Conte ${ }^{1,2}$, Anna Fagotti ${ }^{1,2}$, Giacomo Avesani ${ }^{3}$, Charlotte Trombadori ${ }^{3}$, Alex Federico ${ }^{1}$, \\ Marco D'Indinosante ${ }^{2}$, Maria Teresa Giudice ${ }^{2}$, Silvia Pelligra ${ }^{2}$, Claudio Lodoli ${ }^{4}$, Claudia Marchetti ${ }^{1}$, \\ Gabriella Ferrandina $^{1,2}$, Giovanni Scambia ${ }^{1,2}$, Valerio Gallotta ${ }^{1}$ \\ ${ }^{1}$ Department of Woman, Child and Public Health, Fondazione Policlinico Universitario A. Gemelli IRCCS, Rome, Italy; ${ }^{2}$ Institute of Obstetrics \\ and Gynecology, Università Cattolica del Sacro Cuore, Rome, Italy; ${ }^{3}$ Department of Diagnostic Imaging, Radiation Therapy and Haematology, \\ Fondazione Policlinico Universitario Agostino Gemelli IRCCS, Rome, Italy; ${ }^{4}$ Department of General Surgery, Fondazione Policlinico Universitario \\ A. Gemelli IRCCS, Rome, Italy \\ Contributions: (I) Conception and design: V Gallotta, C Conte; (II) Administrative support: V Gallotta; (III) Provision of study materials or patients: \\ C Conte, V Gallotta; (IV) Collection and assembly of data: C Conte, V Gallotta; (V) Data analysis and interpretation: All authors; (VI) Manuscript \\ writing: All authors; (VII) Final approval of manuscript: All authors. \\ Correspondence to: Dr. Valerio Gallotta, MD. Department of Woman, Child and Public Health, Fondazione Policlinico Universitario A. Gemelli \\ IRCCS, Largo Agostino Gemelli 8, 00168 Rome, Italy. Email: gallottav@gmail.com.
}

\begin{abstract}
The ovarian cancer recurrence occurs in $75 \%$ of patients with advanced FIGO stage, and its treatment is a challenge for the oncologist in gynecology. The standard treatment of recurrent ovarian cancer (ROC) usually includes intravenous chemotherapy according to platinum sensitivity. Furthermore, maintenance treatment with target therapies [e.g., anti-angiogenic drug or PARP inhibitors (PARPi)], should be provided if not precedently administrated. In this scenario, secondary cytoreductive surgery (SCS) remains a practical but controversial option for platinum-sensitive ROC (PSROC). So far, several retrospective series and a Cochrane meta-analysis had concluded that SCS could determine better survival outcomes in ROC with favorable prognostic characteristics, such as the presence of a single anatomical site of recurrence, or when patients are accurately selected for surgery based on complete resection's predictive models. Recently, three randomized clinical trials (RCTs) investigated the role of SCS in PSROC patients selected with different criteria. All the three RCTs showed a significant statistical advantage in progression-free survival (PFS) in the SCS group, with an even more significant difference in patients with complete cytoreduction (about 7-month PFS increased). Data on overall survival (OS) are different in the two completed trials. The GOG213 study has documented a longer OS of PSROC patients who received chemotherapy alone compared to surgery plus chemotherapy. Contrarily, the DESKTOP III trial showed 7.7 months of increased OS in the surgery group $v s$. chemotherapy alone, with a more difference in the complete tumor cytoreduction (CTC) group (12 months). These RCTs thereby suggest that undergoing complete cytoreduction may not be the only key and that the disease biology may also matter. Few recent retrospective series investigated the role of SCS according to BRCA mutation status and the effect of SCS in patients receiving emerging PARPi. A consequence of the developments in SCS and knowledge of different molecular pathways influencing the recurrent disease is that the future research objective should be to individualize and personalize the surgical approach.
\end{abstract}

Keywords: Secondary cytoreduction; recurrent ovarian cancer (ROC); patients' selection; personalized treatment; translational medicine

Submitted Jun 14, 2020. Accepted for publication Feb 07, 2021.

doi: 10.21037/atm-20-4690

View this article at: http://dx.doi.org/10.21037/atm-20-4690 


\section{Introduction}

In 2020 The American Cancer Society estimated that 21,500 women would be diagnosed with epithelial ovarian or fallopian tube cancer, and about $80 \%$ of cases were advanced FIGO stage at diagnosis (1). One of the significant clinical challenges is the treatment of recurrent ovarian cancer (ROC), which occurs in around $75 \%$ of women within 2 years from diagnosis (1).

The standard treatment of ROC patients usually includes intravenous (CHT) chosen based on the platinum sensitivity, determined by the time from last cycle of platinum based CHT and the date of recurrence $(2,3)$. However, this concept has been recently discussed due to the impact of histotype, homologous recombination deficiency (HRD) or status of $B R C A$ genes, previous antiangiogenetic treatment, the pattern of relapse presentation, and other clinical and pathological factors (4-8). Moreover, it also has to be acknowledged that the addition of bevacizumab and PARP inhibitors (PARPi) in the second-line, and very recently, in first-line treatment, would open novel management scenarios of ROC (9-13).

Since 1984, the potential role of secondary cytoreductive surgery (SCS) in ROC patients has been increasingly considered, leading to worldwide acceptance of this treatment option, despite the retrospective design and the heterogeneity of most studies (14-20). A Cochrane metaanalysis had concluded that SCS could determine better survival outcomes in ROC patients, especially platinumsensitive ones, when accurately selected using complete resection's predictive models (21).

In 2019 the first phase III randomized clinical trial (RCT) investigating the role of SCS in first recurrence ovarian cancer was published (22). The patients were randomized to undergo SCS and then receive chemotherapy or to receive chemotherapy alone. This GOG213 trial showed that SCS followed by chemotherapy did not improve OS compared to chemotherapy only (median OS of 50.6 and 64.7 months, respectively; $\mathrm{HR}=1.29, \mathrm{P}=0.08$ ) (22).

However, several considerations emerged from the oncological results in the population enrolled in this RTC: firstly, in the overall study population, $84 \%$ of patients received bevacizumab, and it may have masked an incremental benefit from surgery. The median OS for the entire trial cohort was nearly three times longer than expected when the trial was designed, diluting the treatment effect by enabling a higher likelihood of intervening treatment. As reported by the authors, the precise reasons for these findings are unknown but probably could be related to the introduction of target therapies in the last years (e.g., the use of PARPi).

Second, this RCT did not investigate either the role of $\mathrm{SCS}$ in ROC patients according to the $B R C A$ mutational status or in women who received a new emerging PARPi therapy that has notably improved progression-free survival (PFS).

The second phase III RCT randomized patients to second-line CHT vs. SCS plus CHT (23). Data from the final analysis of DESKTOP III presented at ASCO this year, showed that ROC patients with a positive AGOscore [performance status Eastern Cooperative Oncology Group $(\mathrm{ECOG})=0$, ascites $\leq 500 \mathrm{cc}$, and no residual tumor at initial surgery] who received SCS and CHT resulted in a significantly longer PFS compared with those who received CHT alone (median PFS was 18.4 vs. 14 months, respectively; $\mathrm{HR}=0.66, \mathrm{P}<0.001)$. The primary endpoint analysis showed median overall survival (OS) of 53.7 months with and 46.0 months without surgery $(\mathrm{HR}=0.75,95 \% \mathrm{CI}$ : $0.58-0.96, \mathrm{P}$ value $=0.02)$, with an even more significant difference in patients with complete tumor cytoreduction (CTC) compared to pts without surgery (median OS was 61.9 vs. 46.0 months, HR =0.57, 95\% CI: 0.43-0.76) (23).

In the last ASCO meeting, the preliminary data of another phase III RCT were presented (24). In this SOC1 trial, 356 patients with first platinum-sensitive ROC (PSROC) were selected using a TIAN score combined with a positron emission tomography/computed tomography (PET/CT) image (25). They were randomized to SCS followed by chemotherapy (surgery arm) vs. second-line chemotherapy alone (no surgery arm). The CTC rate was 76.7\%. The co-primary endpoint was PFS and OS. The SCS leads to an advantage of 5.5 months in PFS compared to chemotherapy alone (17.4 vs. 11.9 months, respectively; $\mathrm{HR}=0.58, \mathrm{P}<0.001)$. OS data are immature (24).

In line with the previous RCTs, there is an additional advantage in terms of PFS in the CTC group vs. chemotherapy alone population (19.2 vs. 11.9 months, respectively; $\mathrm{HR}=0.50,95 \% \mathrm{CI}: 0.37-0.66, \mathrm{P}<0.001)$ (Table 1).

This review aims to clarify the role of SCS in an era of personalized medicine and the potential multidisciplinary approach in the treatment of ROC patients.

We present the following article likewise the Narrative Review reporting checklist (available at http://dx.doi. org/10.21037/atm-20-4690). 
Table 1 Secondary cytoreduction in ROC: overview of the literature

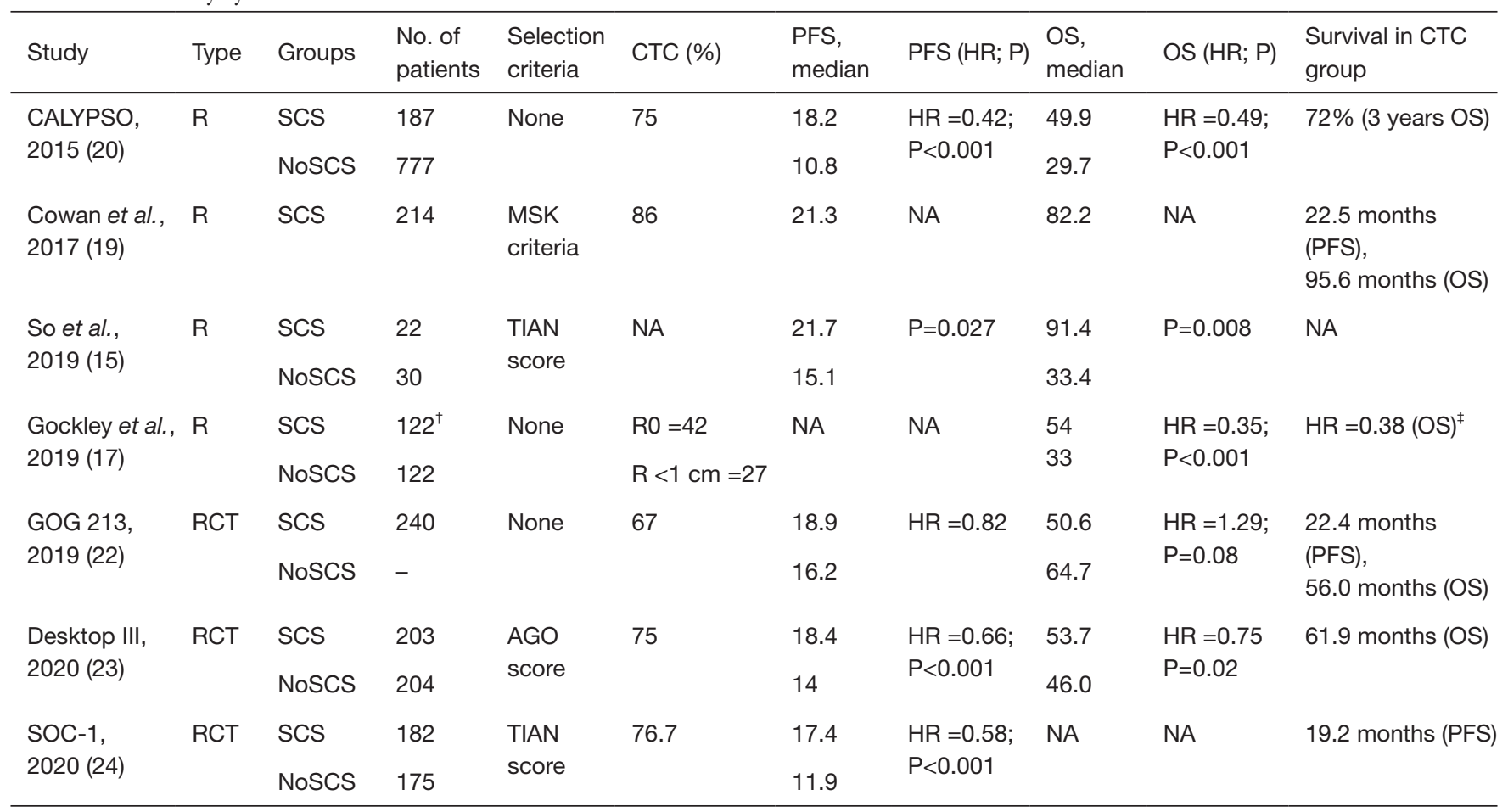

${ }^{\dagger}$, within the propensity score-matched cohort; ${ }^{\ddagger}$, in R0 group vs. NoSCS group. ROC, recurrent ovarian cancer; CTC, complete tumor cytoreduction; PFS, progression-free survival; OS, overall survival; RCT, randomized clinical trial; R, retrospective study; SCS, secondary cytoreductive surgery; NoSCS, chemotherapy alone; MSK, Memorial Sloan Kettering; NA, not available.

\section{Methods}

An enlarged non-systemic literature search of PubMed articles in English with the search item of SCS in ROC was performed. Original articles, RCTs, reviews, meta-analyses, and abstracts presented in conferences on SCS up to May 2020 were included. A narrative synthesis of extracted data was performed and presented using fundamental thematic analysis.

Language restrictions were applied (only articles published in English were considered eligible).

\section{Patient selection}

Development of selection criteria to identify optimal candidates for SCS should be based on prognostic factors, clinical models, and preoperative radiological accurate work-up.

The solitary or localized recurrent disease might be the most useful and intuitive predictors for CTC. Chi et al. in 2006 showed that the median survival time after SCS who had a single-site lesion, multiple lesions, and carcinomatosis were 60,42 , and 28 months, respectively (26). The selection criteria used at the Memorial Sloan Kettering center to identify patients suitable to SCS were the following: patients with a single site of recurrence, regardless of PFS; more than one site of recurrence but without carcinosis and PFS $>12$ months; carcinomatosis and PFS $>30$ months.

Other than CTC after SCS and longer platinum-free interval (PFI) before recurrence diagnosis, several clinical factors drive whether or not to perform SCS.

The score for selection patients suitable to SCS used in the AGO DESKTOP I trial included the following parameters: early FIGO stage at diagnosis or no residual tumor after the first surgery, good performance status, and no ascites at recurrence (27). The CTC was associated with the more prolonged OS than any residual tumor (median OS was 45.2 vs. 19.7 months, respectively; HR $=3.71 ; \mathrm{P}<0.001)$. They showed that the identified criteria panel predicts complete resection in $79 \%$ of patients, and they postulated the relevant impact of preoperative laparoscopic abdominal evaluation to detect the presence of carcinomatosis. Then, the same score was prospectively validated in the DESKTOP II trial (28), confirming the 
validity of this score concerning the positive prediction of CTC in $76 \%$ of AGO score positive women. Therefore, a diagnostic tool such as the AGO score confirms the advantage of a selection process to identify the best candidates for SCS and help avoid unnecessary surgical morbidity. The absence of benefit from SCS because of incomplete tumor resection and the delay induced by surgery in starting indicated chemotherapy can be detrimental to the prognosis of patients.

Afterward, Tian et al. developed a more complex mathematically based model which showed that CTC was correlated with six variables: FIGO stage at diagnosis, residual tumor at first surgery, PFI, ECOG performance status at recurrence, CA-125 level and the presence of ascites at the time of recurrence (25).

Recently, So et al. retrospectively analyzed the benefit of SCS in patients with a low-risk TIAN score. The only variable independently associated with improved OS was SCS. PFS and OS were significantly longer in the SCS group than the CHT group in the matched cohort (median PFS: 21.7 vs. 15.1 months, $\mathrm{P}$ value $=0.027$ and median OS: 91.4 vs. 33.4 months, $\mathrm{P}$ value $=0.008$, respectively) (15).

van de Laar et al., using AGO score and Tian model in a Dutch population-based database with 408 patients, revealed a high positive predictive value for complete SCS, but also relatively high false-negative rates (16). For these reasons, it is essential to underline the role of preoperative radiological work up to indicate localized versus multifocal recurrence, which is one of the most predictor factors of CTC. Moreover, the diagnostic use of laparoscopy could enhance the predictive accuracy of both models (29).

Overall, these scores have different weaknesses to be highlighted: (I) the false-negative rate higher than $50 \%$, which excludes the possibility of SCS for patients in whom surgery could be done successfully; (II) pattern of recurrence and anatomic sites of recurrence were not pre-operatively investigated; (III) no mutational status or administration of target therapy is included in these selection criteria.

\section{The role of imaging in the assessment of ROC}

Considering the increasing complexity of ROC management, it is crucial to have a precise picture of the patient's situation to tailor the best personalized therapy.

The detection of recurrence and its distribution is pivotal to decide the best therapeutic option.

The precise description of recurrence sites, the potential involvement of adjacent organs, and possible anatomical variants drive the oncologic gynecologist in surgical planning, considering possible technical difficulties and potential complications.

Transvaginal ultrasound (TV-US) is usually the firstline imaging method when ROC is clinically suspected due to its wide availability and low cost (30). Nevertheless, it remains inadequate to restage the disease due to its weak capacity in the individuation of peritoneal implants or distant lymphadenopathy and because of its high variability in diagnostic accuracy due to the operator-dependent bias. However, TV-US can precisely detect pelvic recurrence, its relationship with adjacent organs, and the presence of ascites.

CT provides a "roadmap" of the disease distribution and facilitates surgical planning and assessment of the feasibility of optimal SCS (31). There is also emerging evidence that combining anatomical and functional imaging through ${ }^{18}$ F-FDG PET/CT may help evaluate patients with suspected ROC but negative or indeterminate CT findings. In a recent meta-analysis of 34 studies, the pooled area under the curve (AUC) of PET/CT for detecting ROC was significantly higher than that of CT or MRI (32).

${ }^{18} \mathrm{~F}$-FDG PET/CT is the imaging method with the best performance in the detection of ROC (32). ${ }^{18} \mathrm{~F}$-FDG PET/ CT sensitivity and specificity in detecting ROC have been reported in the literature to range between $79 \%$ and $100 \%$ and from $42 \%$ to $100 \%$, respectively (30-35). Gadducci et al. recently described ${ }^{18} \mathrm{~F}$-FDG PET/CT as the most reliable imaging technique for evaluating patients with suspicious ROC, and selecting those more suitable for SCS, with a reported sensitivity of $100 \%$ (36). Such findings are in line with data provided by Cook et al., who stated that the most valuable impact of ${ }^{18} \mathrm{~F}$-FDG PET/CT lies in its use to determine appropriateness for localized treatment approaches, such as SCS or stereotactic body radiotherapy (SBRT) in Oligometastatic Ovarian Cancer (37). Since this investigational tool allows whole-body imaging, distant metastases can be easily detected, in addition to abdominal and pelvic lesions, with an impact on patient management that varies across literature studies, from $44 \%$ to $60 \%$ (30). However, ${ }^{18} \mathrm{~F}$-FDG PET/CT is less accurate in the detection of small foci of the recurrent tumor and military peritoneal involvement, with lower sensitivity than that of laparoscopy or surgical exploration (30). ${ }^{18}$ F-FDG PET/ CT has a low spatial resolution, giving fewer pieces of information in surgical planning.

In a future multimodal approach, ${ }^{18} \mathrm{~F}-\mathrm{FDG} \mathrm{PET} / \mathrm{CT}$ and staging laparoscopy (S-LPS) should be considered 


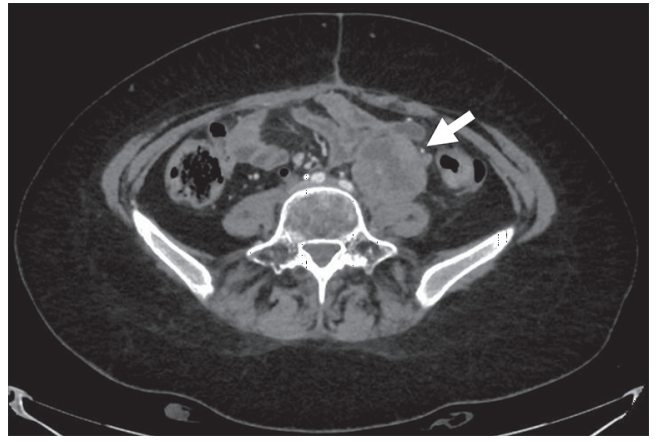

Figure 1 CT portal phase after iodine-contrast injection: an enlarged partially necrotic left common iliac lymph node (arrow) is detected. CT, computed tomography.

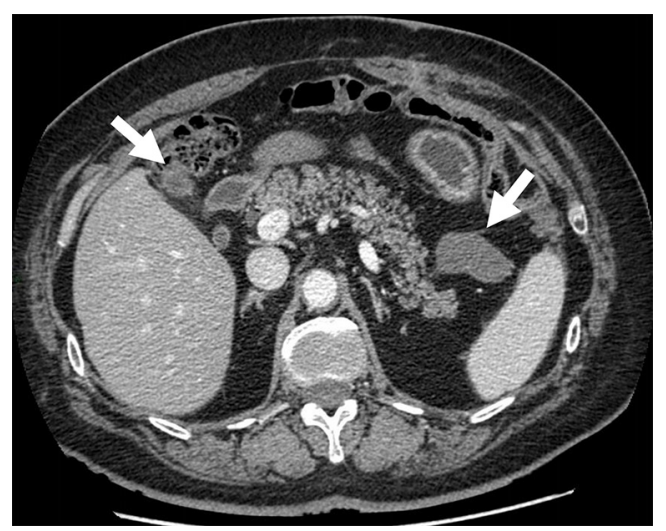

Figure 2 CT portal phase after iodine-contrast injection: two peritoneal implants (arrows) are visible near the spleen and between liver and right colon. CT, computed tomography.

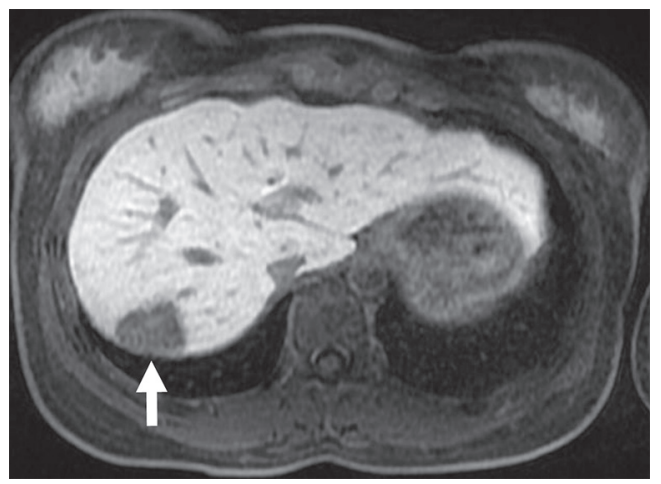

Figure 3 MRI gradient-echo fat-saturated T1-weighted image, delayed hepatobiliary phase after hepatospecific contrast agent injection: the metastasis is clearly seen in the seventh hepatic segment (arrow). complementary because of the potential of each one to identify a different setting of the disease. S-LPS can improve FDG-PET/CT sensitivity versus small nodule carcinosis, as well as the accuracy in the detection of the number of lesions, with an overall accuracy rate of $81.4 \%$ in the combined approach (29).

Contrast-enhanced CT (CE-CT) is usually the technique of choice in the follow-up of patients treated for ovarian cancer, ensuring reproducibility of imaging for comparison (38). However, this method presents some limitations due to its low soft-tissue contrast in evaluating ROC within the pelvis and demonstrating small volume deposits on visceral surfaces (39). Conversely, Wang et al. (40) recently showed that CE-CT might help detect cystic lesions in patients with clinical suspicion of ROC, as ${ }^{18} \mathrm{~F}$-FDG PET/CT has a limited value in detecting cystic lesions in ovarian cancer (41). Furthermore, even if the common sites of ROC include pelvic masses, peritoneal/ pleural metastases, and enlarged lymph nodes (42), some other unusual distant locations may occur, and extendedfield CT is crucial to evaluate adequately potential recurrence. Moreover, CE-CT has an excellent spatial resolution allowing the detailed study of the patient's anatomy and recurrent relationship with adjacent structures, making CT a useful tool for surgical planning (Figures 1,2).

MRI has excellent soft-tissue resolution and the capacity to discriminate between post-treatment changes and tumor recurrence (Figure 3). However, it is used less commonly than other techniques because of its high cost and relatively low availability, but its role is increasing. Scientific evidence reports sensitivity and specificity of $91 \%$ and $86 \%$, respectively (28). Contribution of conventional MRI is limited in small-volume recurrent lesions $(<1 \mathrm{~cm})$ and in sites where the lesions are contiguous to tissues with similar signal intensity (43). Diffusion-weighted magnetic resonance imaging (DWI) sequence showed promising results in detecting limited peritoneal disease and little pathological positive lymph nodes opening new spaces for this technic, alone or complementary to ${ }^{18} \mathrm{~F}-\mathrm{FDG}$ PET/ CT (44). The DWI sequence, together with conventional MRI sequences, raises the accuracy of MRI alone to 84-88\% (27). In addition, Low et al. asserted that ROC shows variable degrees of enhancement after contrast medium administration and that contrast-enhanced MRI is comparable, in sensitivity and specificity, to laparotomy for the detection of ROC (45). 
Emerging technics, like radiomics (the extraction of tissue characteristics of tumor phenotype from images generating features not appreciated by the naked eye) and artificial intelligence, are showing promising results in other malignancies and ovarian cancer $(44,45)$. In the future, radiomics might provide additional information on tumor behavior also in the context of ROC, integrating molecular and genetic findings $(46,47)$.

In conclusion, radiological images play an essential role in depicting ROC, allowing early diagnosis and accurate localization of metastatic disease and guiding the assessment of optimal and personalized treatment, highlighting the importance of a systematic multidisciplinary evaluation of patients with suspected ROC.

\section{Prognostic variables}

In this continuously changing horizon, it is crucial to rethink how ROC patients are classified and selected for SCS or chemotherapy alone, introducing new prognostic variables.

Reliable comparisons of this topic through the existing studies are challenging, considering the differences in the characterization and classification for the pattern of recurrence disease.

Data from the CALYPSO trial documented that SCS was associated with better OS in PSROC, mainly in women with positive prognostic features, such as the presence of a single anatomical site of recurrence (20).

Different patterns of ROC could indicate different biological and clinical characteristics, which raises critical therapeutic questions. We have to consider that the presentation and time of relapse result from the activation of several molecular pathways that drive metastatization by regulating the significant hallmarks of cancer, such as cell adhesion, angiogenesis, apoptosis, and drug resistance. As reported by several previous studies, the anatomic site and the number of relapses seem to impact survival profoundly, with worse oncological outcomes in patients with parenchymal recurrence $(\mathrm{PaR})$ than those with lymph node peritoneal recurrence (PeR) (48-50). Petrillo et al. observed not only an increased survival in case of lymphnodal recurrence (LNR) but also relevant PFS differences between women with $\mathrm{PeR}$ and $\mathrm{PaR}$ (median PFS of 63 months in LNR, 41 months in PeR and 24 months in $\mathrm{PaR}, \mathrm{P}$ value $=0.001)(48)$.

LNRs consist of $12-37 \%$ of ROC, but isolated lymph node relapse (ILNR) is an infrequent event (about
$5 \%)(51,52)$. LNRs are characterized by relatively less chemosensitivity, a low growth rate, and a more indolent behavior than PeR and PaR. Consequently, LNRs might be more suitable for SCS than CHT in selected cases $(48,52,53)$.

It would be clinically relevant to find clinic-pathological variables able to identify patients that would benefit the most from salvage lymphadenectomy (SL). However, in the absence of RCTs due to the rarity of this pattern of disease, we can refer only to the existing retrospective small sample series (48,52,54-56). In an extensive single-institution study, the SL showed a potential therapeutically option in selected PSROC patients with ILNR (57). In the whole series, the median post salvage lymphadenectomy PFS (PSL-PFS) was 21 months, and the 3 -year PSL-PFS was about 37\%, with a better prognosis for patients with ILNR compared to patients with ILNR plus other sites of disease (P value $=0.003)$. At multivariate analysis the PFI $>12$ months and the presence of less than three metastatic lymph-nodes maintained their independent favorable prognostic role for PSL-PFS (57).

Another challenging clinical question is the treatment of liver relapse and to understand the role of SCS in this setting of patients. Several retrospective series have been published assessing that hepatic resection (HR) can be performed within SCS procedures with tolerable surgical complications (58-60).

Recently, in one of the largest series focused on the role of liver resection in platinum sensitivity ROC, the authors reported a median PFS of 35 months and a 3-year PFS of about $49 \%$, a result in line with previous studies. Moreover, it is shown that the BRCA gene mutation(s) is associated with higher PFS after hepatic resection, thus providing a molecularly based line of evidence helping to choose the therapeutic approach to this peculiar clinical setting of ROC patients (61).

Recently, Gockley et al. (17) analyzed the oncological outcomes in a retrospective study that included 626 women with ROC; $23 \%$ of them underwent SCS, and $77 \%$ received CHT. In the propensity score-matched group $(n=244)$, the authors reported a median OS of 54 and 33 months in the SCS and CHT group, respectively (P value $<0.001)$. However, the authors documented that patients who underwent SCS were younger ( $\mathrm{P}$ value $=0.001)$, with an earlier stage disease at diagnosis $(\mathrm{P}$ value $=0.002)$ and higher PFS (P value <0.001), compared with those taking chemotherapy alone. Moreover, in this study, the worse survival outcomes in ROC patients who received 
chemotherapy could probably be related to the presence of higher rates of ascites, more multifocal recurrences sites, or carcinomatosis compared to patients submitted to SCS (17).

Despite several retrospective series showed the benefit of SCS in selected patients with favorable prognostic factors, all the data available are based on non-randomized trials, and multivariate analysis to adjust for confounding factors could reduce but not delete selection bias.

In this context, additional variables influencing the natural history of ovarian cancer might have a significant prognostic role, such as platinum sensitivity, administration of target therapy, and molecular characteristics. Indeed, recent studies showed that $B R C A$ mutation provides a subgroup of ovarian cancer (OC) patients with higher survival rates than wild-type counterparts.

\section{Target therapies}

Target therapies, tumor profiling, and genetic signatures are rapidly evolving the clinical features of ovarian cancer. In the last years, several studies investigate its role and impact on oncological outcomes for ROC patients.

The landscape of treatment options for ROC patients is rapidly changing. It is quite intuitive that in the next future, ROC patients will have more different clinical characteristics from those investigated recent literature. With the introduction of bevacizumab and the increasing rate of PARPi administered to women in the front line as maintenance therapy $(12,13,62,63)$, we could assist in the different biological behavior of the recurrent disease (64).

Petrillo et al., in a case-control study including 222 advanced ovarian cancer (AOC) patients, investigated the pattern and timing of relapse and duration of response to second-line CHT in OC patients treated with first-line CHT with or without bevacizumab (65). They showed longer 7 months PFI in patients who receive standard therapy with bevacizumab $(\mathrm{P}$ value $=0.001)$. Moreover, the patients who use antiangiogenic therapy showed more frequent recurrence in multiple anatomic sites $(\mathrm{P}$ value $=0.035)$ or diffuse PeR ( $\mathrm{P}$ value $=0.046)$.

In the next future, it would be necessary to detect useful molecular features able to predict the efficacy of bevacizumab in the first-line treatment (66). Moreover, it will be crucial to understand the mechanisms of and overcoming adaptive resistance to anti-angiogenesis drugs.

A post-hoc analysis of the SOLO-1 trial presented at ASCO 2019 has shown a significantly increased favorable effect of PARPi (olaparib) in BRCA mutated women without residual disease at first surgery. Consequently, increasing the rate of platinum sensitivity ROC patients and particularly the number of patients with PFI $>24$ months will also be higher the candidate patients to SCS (67).

On the other hand, it is reasonable that the first PSROC after PARPi treatment could show unexpected metastatic sites of disease which could require new medical or surgical strategy.

Regarding the recurrence setting of OC patients, PARPi maintenance therapy after platinum retreatment has been determined to extend secondary PFS in BRCA1/2 mutated patients (BRCAmut) $(10,11)$.

The last data of the SOLO2 trial presented at the last ASCO meeting confirmed these favorable results in prolonging the OS in this setting of patients. Indeed, SOLO2 is the first trial of maintenance PARPi therapy to report OS data from women with PSROC and a BRCAmut (68). In this trial, the patients with PSROC and a $B R C A$ mutation who had received $\geq 2$ lines of treatment were randomized to receive maintenance olaparib or placebo. The final results showed that the maintenance olaparib provided an unprecedented improvement of 12.9 months in median OS vs. placebo (median OS of 51.7 vs. 38.8 months, respectively; $\mathrm{HR}=0.74 ; \mathrm{P}=0.0537$ ) (68).

Some retrospective series recently investigated the SCS efficacy in a heterogeneous population of $B R C A$ mutated and wild-type PSROC women $(57,61,69,70)$.

Marchetti et al., in a retrospective study including 126 patients with ROC, analyzed the correlation between $B R C A$ mutation status and recurrent disease presentation, treatment approach at relapse, and post-recurrence survival (PRS), to identify an effective tailored treatment in this setting of patients (69). Overall, $40 \%$ of patients were BRCA1/2 germline mutation carriers, and $60 \%$ of patients were BRCA wild-type (BRCAwt). In the BRCAmut group, $74 \%$ and $26 \%$ of patients presented with $B R C A 1$ and BRCA2 mutation. The authors found no differences concerning patterns of disease tumor presentation between BRCAmut and BRCAwt women. Women with BRCA1/2 mutation had the best PRS regardless of undergoing secondary cytoreduction or not (5-yr PRS of $73 \%$ vs. $78 \%$, respectively; $\mathrm{P}=0.558)$. Contrarily, BRCAwt women who underwent complete SCS had a significantly longer PRS than BRCAwt women who did not undergo SCS (5-yr PRS of $54 \%$ vs. $42 \%$; P value $=0.048$ ). No residual tumor at SCS was the only independent prognostic factor influencing PRS at multivariate analysis $(\mathrm{OR}=2.25, \mathrm{P}$ value $=0.04) . B R C A$ status, surgical approach at diagnosis (interval debulking 
surgery $v s$. primary debulking surgery), and platinumsensitivity were not found to be prognostic (69).

They suggested that BRCAmut women have the best prognosis regardless of SCS, whereas BRCAwt patients can increase their PRS when CTC is achieved.

The same authors compared SCS plus chemotherapy to chemotherapy alone, selecting only BRCAmut PSROC who received olaparib maintenance after ROC diagnosis (70).

Twenty-three (50\%) BRCAmut women underwent SCS followed by CHT and olaparib maintenance and they were matched with 23 (50\%) BRCAmut patients who received CHT and olaparib. The women in the SCS group had a 3 -year OS of $79 \%$ vs. $42 \%$ in the chemotherapy alone group ( $\mathrm{P}$ value $=0.02)$. The median time to first subsequent therapy (TFST) was significantly longer in the SCS group than in the control group (42 vs. 16 months, respectively; $\mathrm{P}$ value $=0.05)(70)$.

These studies provide a new perspective of view and knowledge of survival after SCS in this setting of patients. All these findings are indicative of biologic heterogeneity in ROC patients. Future research might show clinicpathological and genetic signatures influencing survival outcomes, possibly opening the way for prognosis stratification, target therapies, and personalized surgical treatment.

\section{Minimally invasive surgery (MIS)}

In the last years, MIS techniques have been increasingly used in gynecological oncology practice considering they provide several benefits respect to the open approach: improved visualization, less blood loss, reduction of analgesics need, decreased morbidity, faster recovery, and shorter time to chemotherapy (56,71-80).

In particular, MIS has been successfully employed in selected ROC patients presenting a lower incidence of morbidities, apparently without compromising their survival (73-75). Different retrospective series showed that the laparoscopic and robotic approaches are feasible and safe for selected ROC patients, particularly in case of localized peritoneal disease, lymph-nodes, or isolated parenchymal lesion $(57,71,72)$.

In literature, the rate of optimal cytoreduction documented is consistent across the studies (about 79-98\%) (56,57,71-80).

These results could be associated with increased surgeon experience and skill with the MIS approach and with the acknowledgment that only CTC provides an oncological advantage, as recently shown by the DESKTOP III trial (23). Also, the increased and more accurate preoperative characterization of ROC by radiological workup (e.g., PET/CT scan), and preoperative/intraoperative ultrasound might optimize the patient's selection suitable for MIS approach.

From an oncological point of view, results on survival outcomes for this approach were in line with the open approach, as reported in the literature. Overall, these series reported 2-year PFS between $40 \%$ to $60 \%$ (71-75). Gallotta et al. showed in univariate analysis of factors influencing the post relapse PFS no difference between the open and MIS for patients submitted to lymphadenectomy at the time of SCS (56).

However, the biology and complexity of surgical procedures might preclude this approach in the absence of RCTs (81).

Only in the setting of interval cytoreductive surgery (IDS), a first international, randomized RCT is ongoing to compare the two different approaches in AOC patients that had a complete or partial response to neoadjuvant $\mathrm{CHT}$ (NACT) and normal levels of CA-125 (82).

In 2019 a meta-analysis of three prospective and three retrospective studies documented that a CTC appears feasible and safe with MIS in selected AOC patients who underwent NACT (83).

The authors did not observe any significant difference between the MIS and open groups in obtaining CTC $(\mathrm{P}=0.43)(83)$.

However, only LANCE trial results will provide an evaluation of the oncologic outcomes of MIS and might modify the standard of care for well-selected patients who underwent IDS.

\section{Hyperthermic intraperitoneal chemotherapy (HIPEC)}

HIPEC after cytoreductive surgery has been widely analyzed in women with peritoneal carcinomatosis from several tumors and advanced epithelial OC, with an improvement in PFS and OS (84-88).

Among the patients with ROC, only one randomized controlled trial has evaluated the effect and safety of HIPEC $(89,90)$. The authors showed an oncological benefit for ROC patients who underwent HIPEC, but there are different limitations regarding the randomization method and the delineation of the endpoints (90).

In a recent metanalysis, HIPEC was associated with 
increased OS (HR $=0.566)$ but not with PFS in ROC patients. However, HIPEC improved PFS in women with residual tumor $\leq 10 \mathrm{~mm}$ or no residual tumor, whereas it improved OS only in case of residual tumors $\leq 10 \mathrm{~mm}$ (91).

In a retrospective case series, Fagotti et al. described statistically significant results regarding the rates of secondary recurrence, the duration of secondary response, and mortality, with disease-free survival of $26 v s .15$ months in the HIPEC and no HIPEC group, respectively (92).

The same group reported that MIS for SCS plus HIPEC is safe concerning the toxicity and postoperative outcomes for single isolated relapse (93). Moreover, they reported that the MIS enhances cisplatin blood absorption in women receiving HIPEC, thus providing a strong rationale for developing further novel strategies of endoscopic intraperitoneal drug administration in ROC patients with locoregional disease $(94,95)$.

The ongoing multicenter RCT in a PSROC setting will provide further evidence (NCT 01539785).

\section{Conclusions}

ROC shows heterogeneity of clinical, pathological, and genetic characteristics.

A consequence of the developments in SCS and knowledge of different molecular pathways influencing recurrent disease is that personalized surgery is now a realness. The forthcoming research trend is to achieve a more accurate, individualized approach. More and more sophisticated tumor-targeting strategies are emerging at a molecular level, and the surgical approach could play an emerging role for SCS in ROC patients. For these reasons, the technology is still evolving, and research should be considering the fascinating impact of a multidisciplinary approach even in this setting of patients.

The SCS will continue to play a significant part in increasing oncological outcomes and reducing the impact of cancer treatments on the quality of life in selected patients.

The forsaking of SCS as a ROC treatment approach due to the fast development in molecular genetics and target therapy such as PARPi and bevacizumab, remains an incredibly long way off. The biological heterogeneity and adaptability of cancer cells might make this scenario very difficult to imagine.

Recent data from the final analysis of the DESKTOP III trial (23) have shown a significantly increasing OS benefit (exceeding 12 months) of SCS in patients achieving absent residual tumor compared to patients without surgery. These results highlight the importance of optimal patient selection and tertiary centers with a high chance of achieving a CTC.

\section{Acknowledgments}

Funding: None

\section{Footnote}

Reporting Checklist: The authors have completed the Narrative Review reporting checklist. Available at http:// dx.doi.org/10.21037/atm-20-4690

Conflicts of Interest: All authors have completed the ICMJE uniform disclosure form (available at http://dx.doi. org/10.21037/atm-20-4690). VG serves as an unpaid editorial board member of Annals of Translational Medicine from Mar 2020 to Feb 2022. The other authors have no conflicts of interest to declare.

Ethical Statement: The authors are accountable for all aspects of the work in ensuring that questions related to the accuracy or integrity of any part of the work are appropriately investigated and resolved.

Open Access Statement: This is an Open Access article distributed in accordance with the Creative Commons Attribution-NonCommercial-NoDerivs 4.0 International License (CC BY-NC-ND 4.0), which permits the noncommercial replication and distribution of the article with the strict proviso that no changes or edits are made and the original work is properly cited (including links to both the formal publication through the relevant DOI and the license). See: https://creativecommons.org/licenses/by-nc-nd/4.0/.

\section{References}

1. Siegel RL, Miller KD, Jemal A. Cancer statistics, 2020. CA Cancer J Clin 2020;70:7-30.

2. Wagner U, Marth C, Largillier R, et al. Final overall survival results of phase III GCIG CALYPSO trial of pegylated liposomal doxorubicin and carboplatin vs paclitaxel and carboplatin in platinum-sensitive ovarian cancer patients. Br J Cancer 2012;107:588-91.

3. Pfisterer J, Plante M, Vergote I, et al. Gemcitabine plus carboplatin compared with carboplatin in patients with platinum-sensitive recurrent ovarian cancer: an intergroup trial of the AGO-OVAR, the NCIC CTG, and the 
EORTC GCG. J Clin Oncol 2006;24;4699-707.

4. Peres LC, Cushing-Haugen KL, Anglesio M, et al. Histotype classification of ovarian carcinoma: A comparison of approaches. Gynecol Oncol 2018;151:53-60.

5. Hollis RL, Churchman M, Gourley C. Distinct implications of different BRCA mutations: efficacy of cytotoxic chemotherapy, PARP inhibition and clinical outcome in ovarian cancer. Onco Targets Ther 2017;10:2539-51.

6. Ferrandina G, Legge F, Salutari V, et al. Impact of pattern of recurrence on clinical outcome of ovarian cancer patients: clinical considerations. Eur J Cancer 2006;42:2296-302.

7. Gallotta V, Ferrandina G, Vizzielli G, et al. Hepatoceliac lymph node involvement in advanced ovarian cancer patients: prognostic role and clinical considerations. Ann Surg Oncol 2017;24:3413-21.

8. Gallotta V, Fanfani F, Vizzielli G, et al. Douglas peritonectomy compared to recto-sigmoid resection in optimally cytoreduced advanced ovarian cancer patients: analysis of morbidity and oncological outcome. Eur J Surg Oncol 2011;37:1085-92.

9. Oza AM, Cibula D, Benzaquen AO, et al. Olaparib combined with chemotherapy for recurrent platinumsensitive ovarian cancer: a randomised phase 2 trial. Lancet Oncol 2015;16:87-97.

10. Pujade-Lauraine E, Ledermann JA, Selle F, et al. Olaparib tablets as maintenance therapy in patients with platinumsensitive, relapsed ovarian cancer and a BRCA1/2 mutation (SOLO2/ENGOT-Ov21): a double-blind, randomised, placebo-controlled, phase 3 trial. Lancet Oncol 2017;18:1274-84.

11. Coleman RL, Oza AM, Lorusso D, et al. Rucaparib maintenance treatment for recurrent ovarian carcinoma after response to platinum therapy (ARIEL3): a randomised, double-blind, placebo-controlled, phase 3 trial. Lancet 2017;390:1949-61.

12. Moore K, Colombo N, Scambia G, et al. Maintenance olaparib in patients with newly diagnosed advanced ovarian cancer. N Engl J Med 2018;379:2495-505.

13. González-Martín A, Pothuri B, Vergote I, et al. Niraparib in patients with newly diagnosed advanced ovarian cancer. N Engl J Med 2019;381:2391-402.

14. Berek JS, Hacker NF, Lagasse LD, et al. Survival of patients following secondary cytoreductive surgery in ovarian cancer. Obstet Gynecol 1983;61:189-93.

15. So M, Miyamoto T, Murakami R, et al. The efficacy of secondary cytoreductive surgery for recurrent ovarian, tubal, or peritoneal cancer in Tian-model low-risk patients. J Gynecol Oncol 2019;30:e100.

16. van de Laar R, Kruitwagen RF, IntHout J, et al. Surgery for Recurrent Epithelial Ovarian Cancer in the Netherlands: A Population-Based Cohort Study. Int J Gynecol Cancer 2016;26:268-75.

17. Gockley A, Melamed A, Cronin A, et al. Outcomes of secondary cytoreductive surgery for patients with platinum-sensitive recurrent ovarian cancer. Am J Obstet Gynecol 2019;221:625.e1-14.

18. Bristow RE, Puri I, Chi DS. Cytoreductive surgery for recurrent ovarian cancer: a meta-analysis. Gynecol Oncol 2009;112:265-74.

19. Cowan RA, Eriksson AGZ, Jaber SM, et al. A comparative analysis of prediction models for complete gross resection in secondary cytoreductive surgery for ovarian cancer. Gynecol Oncol 2017;145:230-5.

20. Lee CK, Lord S, Grunewald T, et al. Impact of secondary cytoreductive surgery on survival in patients with platinum sensitive recurrent ovarian cancer: analysis of the CALYPSO trial. Gynecol Oncol 2015;136:18-24.

21. Al Rawahi T, Lopes AD, Bristow RE, et al. Surgical cytoreduction for recurrent epithelial ovarian cancer. Cochrane Database Syst Rev 2013;2013:CD008765.

22. Coleman RL, Spirtos NM, Enserro D, et al. Secondary surgical cytoreduction for recurrent ovarian cancer. $\mathrm{N}$ Engl J Med 2019;381:1929-39.

23. Du Bois A, Vergote I, Ferron G, et al. Randomized controlled phase III study evaluating the impact of secondary cytoreductive surgery in recurrent ovarian cancer: AGO DESKTOP III/ENGOT-ov20. J Clin Oncol 2020;38:abstr 6000.

24. Zang R, Zhu J, Shi T, et al. A randomized phase III trial of secondary cytoreductive surgery in later recurrent ovarian cancer: SOC1/SGOG-OV2. J Clin Oncol 2020;38:abstr 6001.

25. Tian WJ, Chi DS, Sehouli J, et al. A risk model for secondary cytoreductive surgery in recurrent ovarian cancer: an evidence-based proposal for patient selection. Ann Surg Oncol 2012;19:597-604.

26. Chi DS, McCaughty K, Diaz JP, et al. Guidelines and selection criteria for secondary cytoreductive surgery in patients with recurrent, platinum-sensitive epithelial ovarian carcinoma. Cancer 2006;106:19339.

27. Harter P, du Bois A, Hahmann M, et al. Surgery in recurrent ovarian cancer: the Arbeitsgemeinschaft Gynaekologische Onkologie (AGO) DESKTOP OVAR trial. Ann Surg Oncol 2006;13:1702-10. 
28. Harter P, Sehouli J, Reuss A, et al. Prospective validation study of a predictive score for operability of recurrent ovarian cancer: the Multicenter Intergroup Study DESKTOP II. A project of the AGO Kommission OVAR, AGO Study Group, NOGGO, AGO-Austria, and MITO. Int J Gynecol Cancer 2011;21:289-95.

29. Fagotti A, Fanfani F, Rossitto C, et al. A treatment selection protocol for recurrent ovarian cancer patients: the role of FDG-PET/CT and staging laparoscopy. Oncology 2008;75:152-8.

30. Manganaro L, Gigli S, Antonelli A, et al. Imaging strategy in recurrent ovarian cancer: a practical review. Abdom Radiol (NY) 2019;44:1091-102.

31. Gu P, Pan LL, Wu SQ, et al. CA 125, PET alone, PETCT, CT and MRI in diagnosing recurrent ovarian carcinoma: a systematic review and meta-analysis. Eur J Radiol 2009;71:164-74.

32. Son H, Khan SM, Rahaman J, et al. Role of FDG PET/ $\mathrm{CT}$ in staging of recurrent ovarian cancer. Radiographics 2011;31:569-83.

33. Satoh Y, Ichikawa T, Motosugi U, et al. Diagnosis of peritoneal dissemination: comparison of 18F-FDG PET/ CT, diffusion-weighted MRI, and contrast-enhanced MDCT. AJR Am J Roentgenol 2011;196:447-53.

34. Limei Z, Yong C, Yan X, et al. Accuracy of positron emission tomography/computed tomography in the diagnosis and restaging for recurrent ovarian cancer: a meta-analysis. Int J Gynecol Cancer 2013;23:598-607.

35. Suppiah S, Chang WL, Hassan HA, et al. Systematic review on the accuracy of positron emission tomography/ computed tomography and positron emission tomography/ magnetic resonance imaging in the management of ovarian cancer: is functional information really needed? World J Nucl Med 2017;16:176-85.

36. Gadducci A, Simonetti E, Manca G, et al. Positron emission tomography/computed tomography in platinumsensitive recurrent ovarian cancer: a single-center Italian study. Anticancer Res 2020;40:2191-7.

37. Cook SA, Wilson D, Pourghiasian M, et al. The value of PET-CT in ovarian epithelial carcinoma: a populationbased study in British Columbia, Canada. J Med Imaging Radiat Sci 2020;51:264-70.

38. Forstner R, Sala E, Kinkel K, et al. ESUR guidelines: ovarian cancer staging and follow-up. Eur Radiol 2010;20:2773-80.

39. Nasser S, Lazaridis A, Evangelou M, et al. Correlation of pre-operative CT findings with surgical \& histological tumor dissemination patterns at cytoreduction for primary advanced and relapsed epithelial ovarian cancer: a retrospective evaluation. Gynecol Oncol 2016;143:264-9.

40. Wang PH, Huang YT, Ng KK, et al. Detecting recurrent ovarian cancer: revisit the values of whole-body CT and serum CA 125 levels. Acta Radiol 2019;60:1360-6.

41. Takekuma M, Maeda M, Ozawa T, et al. Positron emission tomography with $18 \mathrm{~F}$-fluoro-2-deoxyglucose for the detection of recurrent ovarian cancer. Int J Clin Oncol 2005;10:177-81.

42. Sahdev A, Hughes JH, Barwick T, et al. Computed tomography features of recurrent ovarian carcinoma according to time to relapse. Acta Radiol 2007;48:1038-44.

43. Sanli Y, Turkmen C, Bakir B, et al. Diagnostic value of PET/CT is similar to that of conventional MRI and even better for detecting small peritoneal implants in patients with recurrent ovarian cancer. Nucl Med Commun 2012;33:509-15.

44. Hameeduddin A, Sahdev A. Diffusion-weighted imaging and dynamic contrast-enhanced MRI in assessing response and recurrent disease in gynaecological malignancies. Cancer Imaging 2015;15:3.

45. Low RN, Duggan B, Barone RM, et al. Treated ovarian cancer: MR imaging, laparotomy reassessment, and serum CA-125 values compared with clinical outcome at 1 year. Radiology 2005;235:918-26.

46. Rizzo S, Botta F, Raimondi S, et al. Radiomics of high-grade serous ovarian cancer: association between quantitative CT features, residual tumour and disease progression within 12 months. Eur Radiol 2018;28:4849-59.

47. Lu H, Arshad M, Thornton A, et al. A mathematicaldescriptor of tumor-mesoscopic-structure from computedtomography images annotates prognostic- and molecularphenotypes of epithelial ovarian cancer. Nat Commun 2019;10:764.

48. Petrillo M, Fagotti A, Ferrandina G, et al. Ovarian cancer patients with localized relapse: clinical outcome and prognostic factors. Gynecol Oncol 2013;131:36-41.

49. Petrillo M, Pedone Anchora L, Tortorella L, et al. Secondary cytoreductive surgery in patients with isolated platinum-resistant recurrent ovarian cancer: a retrospective analysis. Gynecol Oncol 2014;134:257-61.

50. Zang RY, Harter P, Chi DS, et al. Predictors of survival in patients with recurrent ovarian cancer undergoing secondary cytoreductive surgery based on the pooled analysis of an international collaborative cohort. Br J Cancer 2011;105:890-6.

51. Ferrero A, Ditto A, Giorda G, et al. Secondary cytoreductive surgery for isolated lymph node recurrence 
of epithelial ovarian cancer: a multicenter study. Eur J Surg Oncol 2014;40:891-8.

52. Legge F, Petrillo M, Adamo V, et al. Epithelial ovarian cancer relapsing as isolated lymph node disease: natural history and clinical outcome. BMC Cancer 2008;8:367.

53. Kimball RE, Schlaerth JB, Kute TE, et al. Flow cytometric analysis of lymph node metastases in advanced ovarian cancer: clinical and biologic significance. Am J Obstet Gynecol 1997;176:1319-26; discussion 1326-7.

54. Blanchard P, Plantade A, Pagès C, et al. Isolated lymph node relapse of epithelial ovarian carcinoma: outcomes and prognostic factors. Gynecol Oncol 2007;104:41-5.

55. Pergialiotis V, Androutsou A, Papoutsi E, et al. Survival outcomes of ovarian cancer patients treated with secondary cytoreductive surgery for isolated lymph node recurrence: A systematic review of the literature. Int J Surg 2019;69:61-6.

56. Gallotta V, Giudice MT, Conte C, et al. Minimally invasive salvage lymphadenectomy in gynecological cancer patients: a single institution series. Eur J Surg Oncol 2018;44:1568-72.

57. Gallotta V, Bruno M, Conte C, et al. Salvage lymphadenectomy in recurrent ovarian cancer patients: Analysis of clinical outcome and BRCA1/2 gene mutational status. Eur J Surg Oncol 2020;46:1327-33.

58. Pekmezci S, Saribeyoglu K, Aytac E, et al. Surgery for isolated liver metastasis of ovarian cancer. Asian J Surg 2010;33:83-8.

59. Roh HJ, Kim DY, Joo WD, et al. Hepatic resection as part of secondary cytoreductive surgery for recurrent ovarian cancer involving the liver. Arch Gynecol Obstet 2011;284:1223-9.

60. Bacalbasa N, Dima S, Brasoveanu V, et al. Liver resection for ovarian cancer liver metastases as part of cytoreductive surgery is safe and may bring survival benefit. World J Surg Oncol 2015;13:235.

61. Gallotta V, Conte C, D'Indinosante M, et al. Prognostic factors value of germline and somatic brca in patients undergoing surgery for recurrent ovarian cancer with liver metastases. Eur J Surg Oncol 2019;45:2096-102.

62. Perren TJ, Swart AM, Pfisterer J, et al. A phase 3 trial of bevacizumab in ovarian cancer. $\mathrm{N}$ Engl J Med 2011;365:2484-96.

63. Tewari KS, Burger RA, Enserro D, et al. Final overall survival of a randomized trial of bevacizumab for primary treatment of ovarian cancer. J Clin Oncol 2019;37:2317-28.

64. Marchetti C, Rosati A, Scambia G, et al. Secondary cytoreduction in platinum-sensitive recurrent ovarian cancer: are we missing something? Ann Transl Med 2019;7:S372.

65. Petrillo M, Amadio G, Salutari V, et al. Impact of bevacizumab containing first line chemotherapy on recurrent disease in epithelial ovarian cancer: a casecontrol study. Gynecol Oncol 2016;142:231-6.

66. Norquist BM, Brady MF, Harrell MI, et al. Mutations in homologous recombination genes and outcomes in ovarian carcinoma patients in GOG 218: an NRG Oncology/ Gynecologic Oncology Group Study. Clin Cancer Res 2018;24:777-83.

67. Mathews CA, Moore KN, Colombo N, et al. Maintenance olaparib after platinum-based chemotherapy in patients (pts) with newly diagnosed advanced ovarian cancer (OC) and a BRCA mutation (BRCAm): efficacy by surgical and tumor status in the Phase III SOLO1 trial. J Clin Oncol 2019;37:abstr 5541.

68. Poveda A, Floquet A, Ledermann JA, et al. Final overall survival (OS) results from SOLO2/ENGOT-ov21: a phase III trial assessing maintenance olaparib in patients (pts) with platinum-sensitive, relapsed ovarian cancer and a BRCA mutation. J Clin Oncol 2020;38:abstr 6002.

69. Marchetti C, De Leo R, Musella A, et al. BRCA Mutation Status to personalize management of recurrent ovarian cancer: a multicenter Study. Ann Surg Oncol 2018;25:3701-8.

70. Marchetti C, Rosati A, Scaletta G, et al. Secondary cytoreductive surgery in platinum-sensitive recurrent ovarian cancer before olaparib maintenance: Still getting any benefit? A case-control study. Gynecol Oncol 2019;155:400-5.

71. Gallotta V, Conte C, Giudice MT, et al. Secondary laparoscopic cytoreduction in recurrent ovarian cancer: a large, single-institution experience. J Minim Invasive Gynecol 2018;25:644-50.

72. Gallotta V, Fagotti A, Fanfani F, et al. Laparoscopic surgical management of localized recurrent ovarian cancer: a singleinstitution experience. Surg Endosc 2014;28:1808-15.

73. Eriksson AGZ, Graul A, Yu MC, et al. Minimal access surgery compared to laparotomy for secondary surgical cytoreduction in patients with recurrent ovarian carcinoma: perioperative and oncologic outcomes. Gynecol Oncol 2017;146:263-67.

74. Escobar PF, Levinson KL, Magrina J, et al. Feasibility and perioperative outcomes of robotic-assisted surgery in the management of recurrent ovarian cancer: a multiinstitutional study. Gynecol Oncol 2014;134:253 6.

75. Magrina JF, Cetta RL, Chang YH, et al. Analysis of 
secondary cytoreduction for recurrent ovarian cancer by robotics, laparoscopy and laparotomy. Gynecol Oncol 2013;129:336-40.

76. Gallotta V, Federico A, Gaballa K, et al. The role of robotic aortic lymphadenectomy in gynecological cancer: surgical and oncological outcome in a single institution experience. J Surg Oncol 2019;119:355-60.

77. Gallotta V, Ghezzi F, Vizza E, et al. Laparoscopic management of ovarian cancer patients with localized carcinomatosis and lymph node metastases: results of a retrospective multi-institutional series. J Minim Invasive Gynecol 2016;23:590-6.

78. Gallotta V, Conte C, D'Indinosante M, et al. Robotic surgery in elderly and very elderly gynecologic cancer patients. J Minim Invasive Gynecol 2018;25:872-7.

79. Gallotta V, Petrillo M, Conte C, et al. Laparoscopic versus laparotomic surgical staging for early-stage ovarian cancer: a case-control study. J Minim Invasive Gynecol 2016;23:769-74.

80. Gallotta V, Cicero C, Conte C, et al. Robotic versus laparoscopic staging for early ovarian cancer: a casematched control study. J Minim Invasive Gynecol 2017;24:293-8.

81. Knisely A, Gamble CR, St Clair CM, et al. The role of minimally invasive surgery in the care of women with ovarian cancer: a systematic review and meta-analysis. J Minim Invasive Gynecol 2020. [Epub ahead of print]. doi: 10.1016/j.jmig.2020.11.007.

82. Nitecki R, Rauh-Hain JA, Melamed A, et al. Laparoscopic cytoreduction After Neoadjuvant ChEmotherapy (LANCE). Int J Gynecol Cancer 2020;30:1450-4.

83. Cardenas-Goicoechea J, Wang Y, McGorray S, et al. Minimally invasive interval cytoreductive surgery in ovarian cancer: systematic review and meta-analysis. J Robot Surg 2019;13:23-33.

84. Elias D, Lefevre JH, Chevalier J, et al. Complete cytoreductive surgery plus intraperitoneal chemohyperthermia with oxaliplatin for peritoneal carcinomatosis of colorectal origin. J Clin Oncol 2009;27:681-5.

85. Mi DH, Li Z, Yang KH, et al. Surgery combined with intraoperative hyperthermic intraperitoneal chemotherapy (IHIC) for gastric cancer: a systematic review and meta-analysis of randomised controlled trials. Int J Hyperthermia 2013;29:156-67.

86. van Driel WJ, Koole SN, Sikorska K, et al. Hyperthermic intraperitoneal chemotherapy in ovarian cancer. N Engl J Med 2018;378:230-40.
87. Petrillo M, De Iaco P, Cianci S, et al. Long-term survival for platinum-sensitive recurrent ovarian cancer patients treated with secondary cytoreductive surgery plus hyperthermic intraperitoneal chemotherapy (HIPEC). Ann Surg Oncol 2016;23:1660-5.

88. Fagotti A, Costantini B, Vizzielli G, et al. HIPEC in recurrent ovarian cancer patients: morbidity-related treatment and long-term analysis of clinical outcome. Gynecol Oncol 2011;122:221-5.

89. Spiliotis J, Halkia E, Lianos E, et al. Cytoreductive surgery and HIPEC in recurrent epithelial ovarian cancer: a prospective randomized phase III study. Ann Surg Oncol 2015;22:1570-5.

90. Harter P, Reuss A, Sehouli J, et al. Brief report about the role of hyperthermic intraperitoneal chemotherapy in a prospective randomized phase 3 study in recurrent ovarian cancer from Spiliotis et al. Int J Gynecol Cancer 2017;27:246-7.

91. Kim SI, Cho J, Lee EJ, et al. Selection of patients with ovarian cancer who may show survival benefit from hyperthermic intraperitoneal chemotherapy: a systematic review and meta-analysis. Medicine (Baltimore) 2019;98:e18355.

92. Fagotti A, Costantini B, Petrillo M, et al. Cytoreductive surgery plus HIPEC in platinum-sensitive recurrent ovarian cancer patients: a case-control study on survival in patients with two year follow-up. Gynecol Oncol 2012;127:502-5.

93. Fagotti A, Costantini B, Gallotta V, et al. Minimally invasive secondary cytoreduction plus HIPEC versus open surgery plus HIPEC in isolated relapse from ovarian cancer: a retrospective cohort study on perioperative outcomes. J Minim Invasive Gynecol 2015;22:428-32.

94. Fagotti A, Petrillo M, Costantini B, et al. Minimally invasive secondary cytoreduction plus HIPEC for recurrent ovarian cancer: a case series. Gynecol Oncol 2014;132:303-6.

95. Cianci S, Ronsini C, Vizzielli G, et al. Cytoreductive surgery followed by HIPEC repetition for secondary ovarian cancer recurrence. Updates Surg 2019;71:389-94.

Cite this article as: Conte C, Fagotti A, Avesani G, Trombadori C, Federico A, D'Indinosante M, Giudice MT, Pelligra S, Lodoli C, Marchetti C, Ferrandina G, Scambia G, Gallotta V. Update on the secondary cytoreduction in platinumsensitive recurrent ovarian cancer: a narrative review. Ann Transl Med 2021;9(6):510. doi: 10.21037/atm-20-4690 